\title{
Efficacy and safety of novel temperature-controlled radiofrequency ablation system during pulmonary vein isolation in patients with paroxysmal atrial fibrillation: TRAC-AF study
}

\author{
Zdenek Starek ${ }^{1}$, Frantisek Lehar ${ }^{1}$, Petr Neuzil ${ }^{2}$, Jan Petru ${ }^{3}$, Josef Kautzner ${ }^{4}$, Petr Peichl $^{4}$, \\ Jean Albenque ${ }^{5}$, Stéphane Combes ${ }^{5}$, Srinivas Dukkipati ${ }^{6}$, and Vivek Reddy ${ }^{7}$ \\ ${ }^{1}$ International Clinical Research Center \\ ${ }^{2} \mathrm{Na}$ Homolce Hospital \\ ${ }^{3}$ Homolce Hospital \\ ${ }^{4}$ IKEM \\ ${ }^{5}$ Clinique Pasteur \\ ${ }^{6}$ Icahn School of Medicine at Mount Sinai \\ ${ }^{7}$ Mount Sinai School of Medicine
}

August 28, 2020

\begin{abstract}
Aims: Saline-irrigated radiofrequency ablation (RFA) for atrial fibrillation (AF) is limited by the absence of reliable thermal feedback limiting the utility of temperature monitoring for power titration. The DiamondTemp (DT) ablation catheter allow efficient temperature-controlled irrigated ablation. We sought to assess the 1-year clinical safety and efficacy of the DT catheter in treating drug-refractory paroxysmal AF. Methods and results: The TRAC-AF trial (NCT02821351) is a prospective, multicenter $(\mathrm{n}=4)$, single-arm study that enrolled patients with symptomatic, drug-refractory paroxysmal AF. Using the DT catheter, point-by-point ablation was performed around all pulmonary veins (PVs) to achieve PV isolation (PVI). Ablation was performed in a temperature-control mode $(60 \mathrm{oC}$, max $50 \mathrm{~W})$ until the split-tip EGM amplitude decreased by [?]75\%. The primary efficacy endpoints included acute procedural success and freedom from AF at 12 months. A total of 62 patients (age $60.3 \pm 11.4$ years; $60 \%$ male) were evaluated after AF ablation using the DT catheter. The mean fluoroscopy and RF ablation times were 9.4 \pm 6.4 min and 19.8 $\pm 8.6 \mathrm{~min}$, respectively. Acute isolation of all PVs was achieved in $100 \%$ of patients. There were no steam pops and there were not seen any char or caugulum on the catheter tip after ablation. There were very few serious procedure/device-related adverse events including a single case of cardiac tamponade (1.6\%). At 1 year, the freedom from AF was $74.2 \%$. Conclusion: This first in man series demonstrates that temperature-controlled irrigated RFA with the DT catheter is efficient, safe, and effective in the treatment of paroxysmal AF.
\end{abstract}

\section{KEY WORDS}

Paroxysmal atrial fibrillation; pulmonary veins isolation; catheter radiofrequency ablation; DiamondTemp ablation catheter; temperature-controlled irrigated ablation; safety and efficacy

\section{INTRODUCTION}

Atrial fibrillation $(\mathrm{AF})$ is the most common clinically significant arrhythmia. It is a major public health concern affecting an estimated 2.3 million people in North America and 4.5 million people in Europe. It has been projected that the prevalence of AF will increase 2.5 times during the next 50 years in the United States. AF is associated with increased cardiovascular morbidity and mortality and the prevalence increases 
over time due to the aging population and increase in age-specific occurrence of AF . Currently, catheter ablation for AF is a widely recognized treatment in patients with drug refractory arrhythmia. The predominant strategy is isolation of the pulmonary veins (PVI) by circular radiofrequency lesions around their ostia in patients with both paroxysmal and persistent AF supplemented by additional ablations, such as linear lesions in the left atrium or ablation of fractionated atrial potentials . The number of technological improvements is growing. For example single shot ablation catheters, high resolution ablation catheters, contact force-sensing technologies and improved navigation technologies, which are aimed to improve outcomes of ablation procedures. Despite technological innovations, currently point-by-point radiofrequency (RF) ablation remains the most common technique to achieve PVI. Despite significant improvements in catheter ablation strategies to treat $\mathrm{AF}$ in recent years, recurrence rates of $\mathrm{AF}$ after $\mathrm{RF}$ ablation due to electrical reconnections still remains relatively high, ranging from 20-55\%. In the the GAP-AF study, $70 \%$ of patients had PV reconnections at 3 months post-ablation. This may be attributed to lack of durable, contiguous, and transmural lesions. Although the point by point method is principally simple, creating a transmural, continuous lines around the PV ostia may be a major challenge. The major improvement in the ablation technology in the last ten years is technology of irrigated tip catheters. Saline irrigation of the tip of the catheter decreased the incidence of thrombus and char formation on the ablation tip and increase lesion size. However saline irrigation limits the temperature feedback and those catheters are typically operated in power control mode. Overall settings of irrigated catheters can cause inadequate lesion creation during PVI.

DiamondTemp (DT) Cardiac Ablation System (DiamondTemp Catheter, EPIX Therapeutics, Santa Clara, California, USA, formerly known as Advanced Cardiac Therapeutics, which was acquired by Medtronic, Inc. (Dublin, IR) in March 2019), is a recently developed ablation system based on composite-tip, diamondembedded, temperature sensing, saline-irrigated RF ablation catheter with 6 insulated thermocouples on the ablation tip surface which directly measure the tissue surface temperature. The DT catheter allows ablation in temperature control mode with rapid diffusion of heat due to an industrial diamond embeded catheter tip with the potential to fastly create deeper and more transmural lesions. According to animal data, $92.7 \%$ of lesions during ablation of pig's atria were transmural The aim of our work is to describe the first multicenter clinical experience with the DT ablation system during ablation of drug refractory, recurrent, symptomatic paroxysmal AF (TRAC-AF study).

\section{METHODS}

The TRAC-AF (ACT DiamondTemp TempeRAture-Controlled and Contact Sensing RF Ablation Clinical Trial for Atrial Fibrillation) trial is a prospective, multi-center, open-label, single-arm study (NCT02821351) that enrolled patients with symptomatic, drug-refractory paroxysmal AF to undergo PVI using a novel catheter with a diamond embedded tip (DiamondTemp Catheter, formerly Epix Therapeutics, Inc., Sunnyvale, CA, now Medtronic, Inc. (Dublin, IR) in March 2019). The study was approved by the institutional review boards at each of the 4 clinical sites. All participants enrolled in this study provided informed consent. Of the 71 patients enrolled in this study, the acute procedure, 3-month PV remapping, and 6 months outcome results were previously reported for 35 patients enrolled in one center (15).

Key inclusion criteria were: (i) age [?]18 years, (ii) recurrent and symptomatic AF with [?]2 episodes in prior 12 months with at least one episode documented by Holter monitoring, (iii) failure of at least one class I-IV anti-arrhythmic drug (AAD), and (iv) able to provide informed consent. Key exclusion criteria were: (i) prior left atrial ablation, (ii) cardiac surgery within prior two months, (iii) intracardiac thrombus on the day of the procedure by transesophageal echocardiography, (iv) active infection, fever, or sepsis, (v) uncontrolled NYHA class III or IV heart failure, (vi) unable or unwilling to take anticoagulants, (vii) women of childbearing age who are pregnant or not willing to use contraception for the duration of the study, and (viii) life expectancy $<1$ year.

The details of the DiamondTemp (DT) catheter and system have previously been reported (15). Briefly, the DT catheter is $7.5 \mathrm{~F}$ and has a $4.1 \mathrm{~mm}$ composite electrode tip that consists of a two-part platinum-iridium electrode and two industrial grade diamonds that allow the rapid dissipation of heat due to their high thermal diffusivity (Figure 1). The distal aspect of the composite electrode is $0.6 \mathrm{~mm}$ and is electrically insulated 
from the proximal component which allows for high resolution EGM sensing. The rapid heat dissipation allows for a reduced saline irrigation rate of $8 \mathrm{ml} / \mathrm{min}$ through 6 irrigation ports. Three proximal and 3 distal thermocouples allow for accurate estimation of tissue temperature. There are two additional proximal ring electrodes. A custom RF generator (EPIX Therapeutics, Santa Clara, California, USA) delivers RF energy in a temperature-control mode.

All ablation procedures were performed under conscious sedation. Procedures were performed using a threedimensional electroanatomical mapping system (EnSite Velocity, Abbott, Plymouth, Minnesota, USA). Standard double transseptal punctures were performed. The DT catheter was placed within a deflectable sheath (Agilis, Abbott, Plymouth, Minnesota, USA) and 20-pole circular mapping catheter was placed through a second non-deflectable sheath (SL1, Abbot, Plymouth, Minnesota, USA). PVI was performed with standard point-by-point method in a temperature-control mode (limit temperature $60 \mathrm{deg}$, maximum power $50 \mathrm{~W}$ ) until a 75-80\% reduction in the split-tip EGM amplitude was achieved. During ablation on the posterior wall of the left atrium, the target temperature was reduced to $55 \mathrm{degC}$. Saline irrigation rate was $2 \mathrm{ml} / \mathrm{min}$ during mapping and $8 \mathrm{ml} / \mathrm{min}$ during ablation. Ipsilateral $\mathrm{PVs}$ were isolated with a wide encircling lesion set (wide area circumferential antral ablation (WACAA), see Figure 2. Additional ablations were permitted in cases of atrial flutter or other concomitant arrhythmias induced during the procedure.

Following ablation, all patients were discharged on oral anticoagulation for at least 3 months. AADs were discontinued at the discretion of the treating physician. Patients were followed with clinic visits at 3 months, 6 months, and 12 months post-procedure and Holter monitoring was conducted at 3 months and 12 months following the procedure.

At 3 months after the index procedure, subgroup of patients from one center underwent a repeat procedure to assess for PV reconnection, regardless of the intervening symptomatology. During this procedure, the durability of PVI was assessed with a circular mapping catheter. If PV reconnection was identified, the DT catheter was used to ablate the site(s) of electric reconnection to achieve re-isolation (15) Eight reablated patients were excluded from evaluation and total number evaluated patients in this work is 63 .

Endpoints: The primary effectiveness endpoint was (i) acute procedural success defined as electrical isolation of all clinically releveant PVs with demonstration of block or isolation of signals confirmed after ablation, (ii) freedom from AF during 12 months follow up. The primary safety endpoint consisted of nature and frequency of serious adverse events (SAE) and serious adverse device effects (SADE) during the ablation procedure or within 7 days afterwards. Secondary safety outcome consisted of nature and frequency of SAEs and SADEs up to 12 months post-ablation. Adverse events (AEs) were adjudicated by a team of investigators at the particular center.

Statistical analysis: Continuous variables were described as mean+-standard deviation (SD), or median (minimum, maximum).

\section{RESULTS}

A total of 63 patients underwent PVI using the DT catheter and system. The mean age of the patients was $60.3+-11.4$ years; $60 \%$ male (Table 1 ). The mean CHA2DS2-VASc score of the subjects was 1,8 +- 1,2. A total of $77.1 \%$ of patients were on oral anticoagulation, $6.3 \%$ on ASA, and $9.5 \%$ did not take any medication at baseline, for details see Table 2 .

The procedural characteristics are shown in Table 3. Mean fluoroscopy and procedure times were $9.6+-$ $6.6 \mathrm{~min}$ and $155+-47 \mathrm{~min}$, respectively. The mean number of RF applications per patient was $66.6+-$ 24.0 with a mean duration of $17.5+-2.4 \mathrm{sec}$ per RF application. A total of $59 \mathrm{PVs}$ were targeted in the 63 patients. Seven patients (10\%) underwent additional ablation in addition to PVI (four ablation of cavotricuspid isthmus for typical atrial flutter, two ablation of atypical left atrial flutter and one typical atrio-ventricular nodal tachycardia ablation). The primary effectiveness endpoint of acute PVI was achieved in 100\% of targeted PVs. The primary safety endpoint of SAEs or SADEs within 7 days of the procedure occurred in two $(2.9 \%)$ patients. One patient developed a delayed pericardial effusion $8 \mathrm{~h}$ after the procedure 
which was successfully drained and the patient was discharged. A few weeks later, the same patient returned with recurrent pericardial effusion that was again drained. No further accumulation occurred, and the patient did well in follow-up. The exact etiology of this effusion was unclear. During the procedure, there were no instances of audible pops, and at the end of the procedure, the intracardiac echocardiography catheter was used to document the absence of any pericardial effusion. One patient developed after the procedure, a transient ischemic attack (impairment of vision) with complete resolution and no finding on CT of the brain. Seven patients developed AEs, six pseudoaneurysm of femoral vein, one patient had single orthostatic syncope day after procedure. During follow-up, two patients suffered from back pain with complete resolution, see Table 4. Two months following the ablation procedure, 1 patient died; a post-mortem examination indicated that the cause of death was systolic heart failure related to coronary artery disease. This was adjudicated to be unrelated to either the procedure or study catheter.

As previously reported, subgroup of 23 patients from one center were scheduled for a second PV remapping procedure at $\sim 3$ months. Out of those, 8 patients underwent reisolation of PVs due to reconnections (15). These eight reablated patients were excluded from the evaluation of 12-month efficacy. In the surviving 62 patients (without one deceased patient) the freedom from AF at 12 months was $74.2 \%$. Of the whole group of 62 patients 29 patients were totally without antiarrhytmic therapy and 18 were remaining on class I or class III AADs. A total of 25 patients used betablocker, for details see Table 2.

\section{DISCUSSION}

Point-by-point creation of the ablation lines using externally irrigated ablation catheters in power control mode during PVI remain the standard current procedure through its flexibility and ability to perform extra PV ablation. However, there is still demand for improvement of technologies to make procedure faster and lesions more durable. Introduction of a new ablation system with the possibility of temperature control mode should overcome limitations of standard irrigated tip catheters, lead to deeper and more permanent lesions, and reduce the incidence of the chronic reconnections. The DT Cardiac Ablation System has several features for improved creation of the ablation lesions. The diamond embedded tip of the catheter with very rapid heat diffusion allows for creation of faster lesions and reduced irrigation to $8 \mathrm{ml} / \mathrm{min}$. Reduced irrigation with a complex system of surface thermocouples allows the use of temperature control mode with better titration of energy during ablation with variable stability and contact force and subsequently creates lesions more efficiently, reliably, and safely. The composite, dual part tip of the DT catheter enables sensing of high-resolution EGMs, which might be better for monitoring of lesion creation and minimizing collateral injury. It is expected, that ablation with a novel system will be faster and safer with minimizing of pops or char formation.

The TRAC-AF study clearly shows, that despite short RF application time in the majority of patients, durable PVI was achieved with a longterm success rate comparable to patients ablated with standard irrigated tip catheters. Our study confirmed the pilot results of Iwasawa et al with a larger group of patients. We show that temperature-controlled, saline-irrigated RF ablation with the DT Cardiac Ablation System equipped with an array of thermocouples at the tip-tissue interface is very efficient and safe with a high probability of achieving transmural atrial lesions. Preclinical experimental data show that a $75 \%$ reduction in voltage was achieved with $13.3+-6.0 \mathrm{~s}$ of RF, and complete lesion transmurality was achieved in $92.7 \%$ of lesions .

A significantly lower total radiofrequency ablation time was seen with the DT catheter compared to AF patients ablated with standard irrigation catheters in other contemporary paroxysmal AF ablation studies, such as the Fire and Ice Study (Cryoballoon or Radiofrequency Ablation for Paroxysmal Atrial Fibrillation) and the Heartlight (CardioFocus, Marlborough, Massachusetts) multicenter clinical trials, see Figure 3.

Total fluoroscopy time was significantly shorter in the DT group as well $(9.6+-6.6$ min for TRACK AF patients, 16,6 +- 17,8 min. and $29.7+-21.0$ min for Fire and Ice Study and Heartlight resp.), which is well-aligned with the trend to decrease radiation exposure which has been dramatically decreasing over the last decade for PVI. With older technology we exploited our chances to decrease exposure through operator experience, annual case volume, and technology evolution . Contact force catheters are thought 
to be major contributor to the trend, especially in more lengthy procedures for persistent AF . Inclusion of other modalities in the ablation strategy, such as pre-procedural computed tomography and 3D rotational angiography, has also contributed to lower radiation exposure (11).

DT technology thus further allows to decrease exposure times for patients and attending physicians, further decrease would be possible only by fluorless procedures .

The 12-month rate of freedom from $\mathrm{AF}$ of $72.5 \%$ is similar to the success rates of catheter ablations with standard irrigated tip catheters , , . This data support noninferiority of the new ablation system regarding efficacy.

From a safety view, the DT catheter seems to be as safe as standard contact force ablation catheters. There were no audible pops, or char formation. There was only one delayed pericardial effusion related to the ablation procedure. Rate of complications nonrelated or possibly related to the investigational device was $4.3 \%$ and was comparable with complication rates during catheter ablation with standard irrigated tip catheters

The presented study has several limitations. Our project is the first larger study to deal with the new DT ablation system. The number of patients is limited and the study has nonrandomized, single-arm design. Comparison with other ablation studies is indirect and for a more definitive confirmation of efficacy and safety, a randomized, large study comparing this system with standard irrigated contact force catheters is needed. Such a study which recruited patients with paroxysmal AF has been recently completed and is to be published.

\section{Conclusions}

The TRAC AF study demonstrated that temperature-controlled, irrigated RF ablation using DiamondTemp Cardiac Ablation System is efficient and safe, and allows the rapid creation of durable lesions. Total ablation time appears to be shorter in comparison with standard technology of power-controlled, irrigated ablation using contact force sensor catheters.

\section{Group Members}

Jiri Jez, MD ${ }^{1,2}$

Martin Pesl, MD, $\mathrm{PhD}^{1,2,3}$

Lucie Sediva, MD ${ }^{4}$

Libor Dujka MD ${ }^{4}$

Moritoshi Funasako MD ${ }^{4}$

Bashar Aldhoon $\mathrm{MD}^{5}$

Serge Boveda MD ${ }^{7,8}$

\section{Figures}

Figure 1. DiamondTemp Catheter - the temperature-controlled RF ablation catheter contains 6 thermocouples that are equally, radially spaced and a diamond shunt network to facilitate the rapid conduction of thermal energy.

Figure 2. 3D electroanatomical map of the left atrium with PVI lesion set. Ipsilateral PV pairs were isolated with a single wide-area circumferential antral ablation (WACAA) lesions. Red dots - left PVs WACAA, blue dots - right PVs WACAA, yellow dots - the place of isolation of individual pair of PVs.

Figure 3 Graphical comparison of the total ablation time of the TRAC-AF (ACT DiamondTemp Temperature-Controlled and Contact Sensing RF Ablation Clinical Trial for Atrial Fibrillation) study and paroxysmal atrial fibrillation studies using standard irrigated contact force catheters (the TactiCath arm 
of the TOCCASTAR (TactiCath Contact Force Ablation Catheter Study for Atrial Fibrillation), and the Thermocool arm of the Heartlight clinical trial). PVI = pulmonary vein isolation; $\mathrm{RF}=$ radiofrequency.

\section{TABLES}

Table 1 Baseline Clinical Characteristics $(\mathrm{n}=63)$

Table 2 Drug management $(\mathrm{n}=63)$

Table 3 Procedure Data $(\mathrm{n}=63)$

Table 4 Safety Endpoints $(\mathrm{n}=63)$

\section{References}

1. Feinberg WM, Blackshear JL, Laupacis A, et al. Prevalence, age distribution, and gender of patients with atrial fibrillation. Analysis and implications. Arch Intern Med 1995;155:469-473.

2. Go AS, Hylek EM, Phillips KA, et al. Prevalence of diagnosed atrial fibrillation in adults: national implications for rhythm management and stroke prevention: the AnTicoagulation and Risk Factors in Atrial Fibrillation (ATRIA) Study. JAMA 2001;285:2370-2375.

3. Krahn AD, Manfreda J, Tate RB, et al. The natural history of atrial fibrillation: incidence, risk factors, and prognosis in the Manitoba Follow-Up Study. Am J Med 1995;98:476-484.

4. Calkins H, Kuck KH, Cappato R, et al. 2012 HRS/EHRA/ECAS expert consensus statement on catheter and surgical ablation of atrial fibrillation: recommendations for patient selection, procedural techniques, patient management and follow-up, definitions, endpoints, and research trial design. J Interv Card Electrophysiol 2012;33:171-257.

5. Haissaguerre M, Jais P, Shah DC, et al. Spontaneous initiation of atrial fibrillation by ectopic beats originating in the pulmonary veins. N Engl J Med 1998;339:659-666.

6. Haissaguerre M, Jais P, Shah DC, et al. Right and left atrial radiofrequency catheter therapy of paroxysmal atrial fibrillation. J Cardiovasc Electrophysiol 1996;7:1132-1144.

7. Nademanee K, McKenzie J, Kosar E, et al. A new approach for catheter ablation of atrial fibrillation: mapping of the electrophysiologic substrate. J Am Coll Cardiol 2004;43:2044-2053.

8. Luik A, Kunzmann K, Hormann P, et al. Cryoballoon vs. open irrigated radiofrequency ablation for paroxysmal atrial fibrillation: long-term FreezeAF outcomes. BMC Cardiovasc Disord 2017;17:135.

9. Kuck KH, Reddy VY, Schmidt B, et al. A novel radiofrequency ablation catheter using contact force sensing: Toccata study. Heart Rhythm 2012;9:18-23.

10. Starek Z, Lehar F, Jez J, et al. Periprocedural 3D imaging of the left atrium and esophagus: comparison of different protocols of 3D rotational angiography of the left atrium and esophagus in group of 547 consecutive patients undergoing catheter ablation of the complex atrial arrhythmias. Int J Cardiovasc Imaging 2016;32:1011-1019.

11. Starek Z, Lehar F, Jež J, et al. 3D X-ray imaging methods in support catheter ablations of cardiac arrhythmias. Int J Cardiovasc Imaging. 2014;30:1207-1223.

12. Neuzil P, Reddy VY, Kautzner J, et al. Electrical reconnection after pulmonary vein isolation is contingent on contact force during initial treatment: results from the EFFICAS I study. Circ Arrhythm Electrophysiol 2013;6:327-333.

13. Kuck KH, Hoffmann BA, Ernst S, et al. Impact of Complete Versus Incomplete Circumferential Lines Around the Pulmonary Veins During Catheter Ablation of Paroxysmal Atrial Fibrillation: Results From the Gap-Atrial Fibrillation-German Atrial Fibrillation Competence Network 1 Trial. Circ Arrhythm Electrophysiol 2016;9:e003337. 
14. Afzal MR, Chatta J, Samanta A, et al. Use of Contact Force Sensing Technology During Radiofrequency Ablation Reduces Recurrence of Atrial Fibrillation: A Systematic Review and Meta-Analysis. Heart Rhythm 2015;12:1990-1996.

15. Iwasawa J, Koruth JS, Petru J, et al. Temperature-Controlled Radiofrequency Ablation for Pulmonary Vein Isolation in Patients With Atrial Fibrillation. J Am Coll Cardiol 2017;70:542-553.

16. Kuck KH, Brugada J, Fürnkranz A, et al. Cryoballoon or Radiofrequency Ablation for Paroxysmal Atrial Fibrillation. N Engl J Med 2016;374:2235-2245.

17. Reddy VY, Dukkipati SR, Neuzil P, et al. Randomized, controlled trial of the safety and effectiveness of a contact force-sensing irrigated catheter for ablation of paroxysmal atrial fibrillation: results of the TactiCath Contact Force Ablation Catheter Study for Atrial Fibrillation (TOCCASTAR) study. Circulation 2015;132:907-915.

18. Voskoboinik A, Kalman ES, Savicky Y, et al. Reduction in radiation dose for atrial fibrillation ablation over time: A 12-year single-center experience of 2344 patients. Heart Rhythm 2017;14:810-816.

19. Naniwadekar A, Joshi K, Greenspan A, et al. Use of the new contact force sensing ablation catheter dramatically reduces fluoroscopy time during atrial fibrillation ablation procedures. Indian Pacing Electrophysiol J 2016;16:83-87.

20. Casella M, Dello Russo A, Pelargonio G, et al. Near zerO fluoroscopic exPosure during catheter ablAtion of supRavenTricular arrhYthmias: the NO-PARTY multicentre randomized trial. Europace 2016;18:15651572 .

21. Dukkipati SR, Cuoco P, Kutinsky I, et al. Pulmonary vein isolation using the visually guided laser balloon: a prospective, multicenter, and randomized comparison to standard radiofrequency ablation. J Am Coll Cardiol 2015;66:1350-1360.

22. Cappato R, Calkins H,Chen SA, et al. Updated Worldwide Survey on the Methods, Efficacy, and Safety of Catheter Ablation for Human Atrial Fibrillation. Circ Arrhythm Electrophysiol 2010;3:32-38.

\section{TABLES}

Table 1 Baseline Clinical Characteristics $(\mathrm{n}=63)$

Table 1 Baseline Clinical Characteristics $(\mathrm{n}=63)$

Age (yrs)

Male

LVEF (\%)

LA diameter $(\mathrm{mm})$

Height $(\mathrm{cm})$

Weight $(\mathrm{kg})$

Resting Heart Rate (bpm)

Resting Systolic Blood Pressure ( $\mathrm{mmHg}$ )

Resting Diastolic Blood Pressure ( $\mathrm{mmHg}$ )

Hemoglobin $(\mathrm{g} / \mathrm{l})$

Creatinine $(\mu \mathrm{mol} / \mathrm{l})$

Glomerular Filtration Rate $\left(\mathrm{ml} / \mathrm{min} / 1.73 \mathrm{~m}^{2}\right)$

AF duration (months)

Hypertension

Heart failure

Diabetes mellitus

Stroke

CAD 
Table 1 Baseline Clinical Characteristics $(\mathrm{n}=63)$

CHA2DS2-VASc

Antiarrhythmic Therapy

Class I

Class II

Class III

Class IV

Anticoagulation/Antiagregation Therapy

Vitamin K Antagonist

Direct Oral Anticoagulants

Acetylsalicylic Acid

Values are mean $\pm \mathrm{SD}$ or $\mathrm{n}(\%) ; \mathrm{AF}=$ atrial fibrillation; $\mathrm{CAD}=$ coronary artery disease; $\mathrm{LA}=$ left atrial; $\mathrm{LVEF}=$ left ver

Table 2 Drug management $(\mathrm{n}=63)$

Table 2 Drug management $\left(\mathrm{n}=63^{*}\right)$

Table 2 Drug management $\left(\mathrm{n}=63^{*}\right)$

Baseline

$\mathrm{n}=63$

Antiarrhythmic Therapy

Class I

$33(52.4)$

Class II

$26(41.3)$

Class III

$14(22.2)$

Class IV

$0(0)$

Without Therapy

$10(15.9)$

Anticoagulation/Antiagregation Therapy

Vitamin K Antagonist

$17(27)$

Direct Oral Anticoagulants

$36(57.1)$

Acetylsalicylic Acid

$4(6.3)$

Without Therapy

$6(9.5)$

Missing event data from subject who died before $6 \mathrm{M} \mathrm{FU}$, values are n (\%)

* Missing event data from subject who died be

Table 3 Procedure Data $(\mathrm{n}=63)$

\begin{tabular}{ll}
\hline Table 3 Procedure Data $(\mathrm{n}=63)$ & Table 3 Procedure Data $(\mathrm{n}=63)$ \\
\hline No. of RF Applications Per Patient & $66.6 \pm 24.0$ \\
Ablation Duration $(\mathrm{sec})$ & $17.5 \pm 2.4$ \\
Average Power $(\mathrm{W})$ & $36.2 \pm 2.7$ \\
Max Power $(\mathrm{W})$ & $50.8 \pm 0.5$ \\
Temperature Set-Point $\left({ }^{\circ} \mathrm{C}\right)$ & $58.1 \pm 1.6$ \\
Max Temperature $\left({ }^{\circ} \mathrm{C}\right)$ & $65.0 \pm 3.5$ \\
Average Temperature $\left({ }^{\circ} \mathrm{C}\right)$ & $48.2 \pm 1.9$ \\
Max Impedance () & $134.7 \pm 31.2$ \\
Average Impedance () & $95.5 \pm 8.9$ \\
Total RF Ablation Time $(\min )$ & $19.8 \pm 8.6$ \\
Total Procedure Time $(\min )$ & $155 \pm 47$ \\
Total Fluoroscopy Time $(\operatorname{mins})$ & $9.6 \pm 6.6$ \\
Total irrigated volume used for cooling $(\mathrm{ml})$ & $322.6 \pm 99$ \\
Values are mean \pm SD. & Values are mean $\pm \mathrm{SD}$. \\
\hline
\end{tabular}


Table 4 Safety Endpoints $(\mathrm{n}=63)$

\begin{tabular}{lll}
\hline Table 4 Safety Endpoints $(\mathrm{n}=63)$ & Table 4 Safety Endpoints $(\mathrm{n}=63)$ & Table 4 Safety Endpoints $(\mathrm{n}=63)$ \\
\hline & SAE & $\mathrm{AE}$ \\
$\begin{array}{l}\text { Primary Safety } \\
\text { (Procedure through } 7 \mathrm{~d})\end{array}$ & $2(2.9)$ & $4(5.7)$ \\
$\begin{array}{l}\text { Long Term Safety } \\
(7 \mathrm{~d} \text { through 30d) }\end{array}$ & $1(1.4)$ & $0(0)$ \\
$\begin{array}{l}\text { Chronic Safety (30d-12M) } \\
\text { Values are n (\%) }\end{array}$ & $0(0)$ & $2(2.9)$ \\
\hline
\end{tabular}
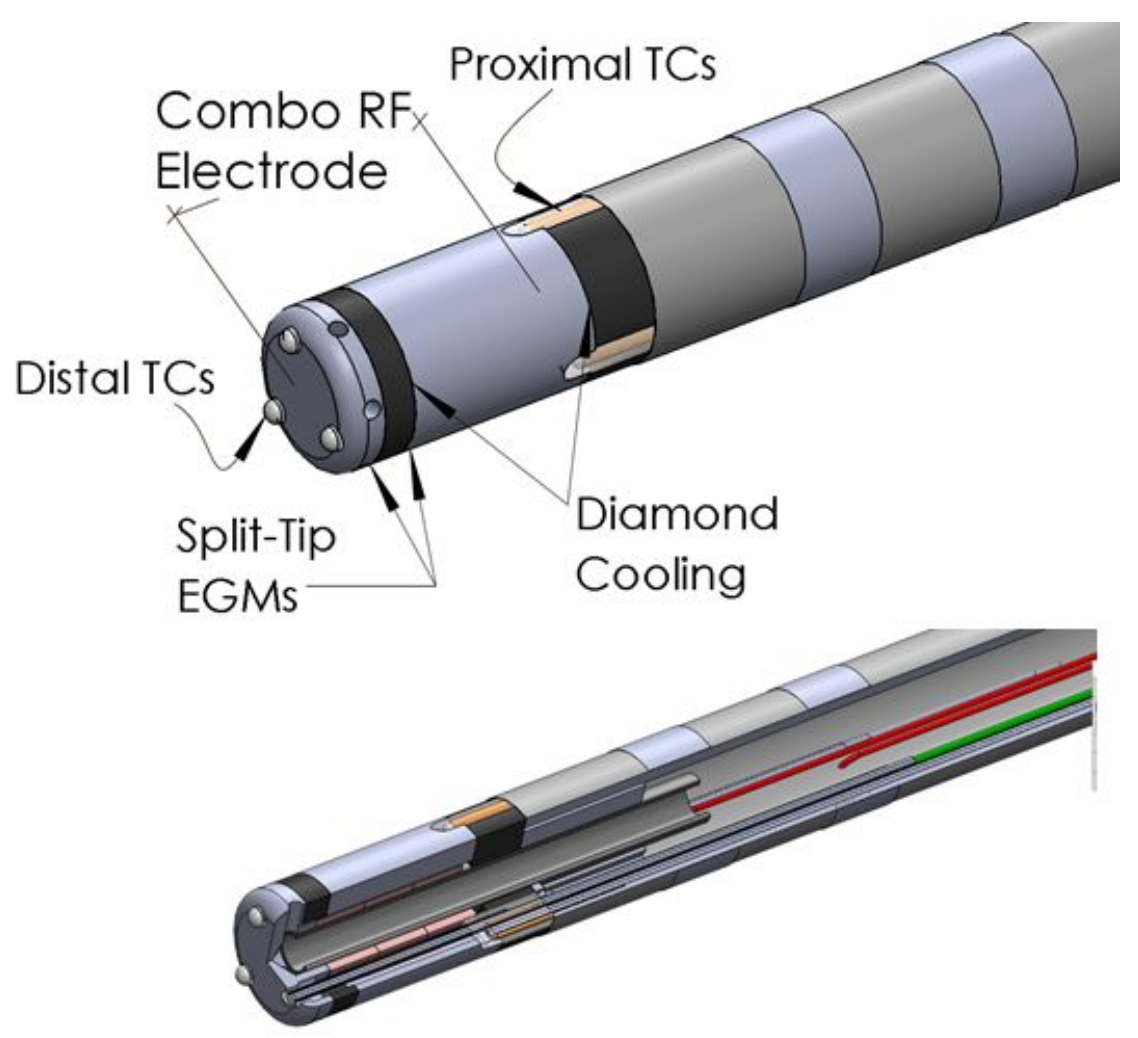


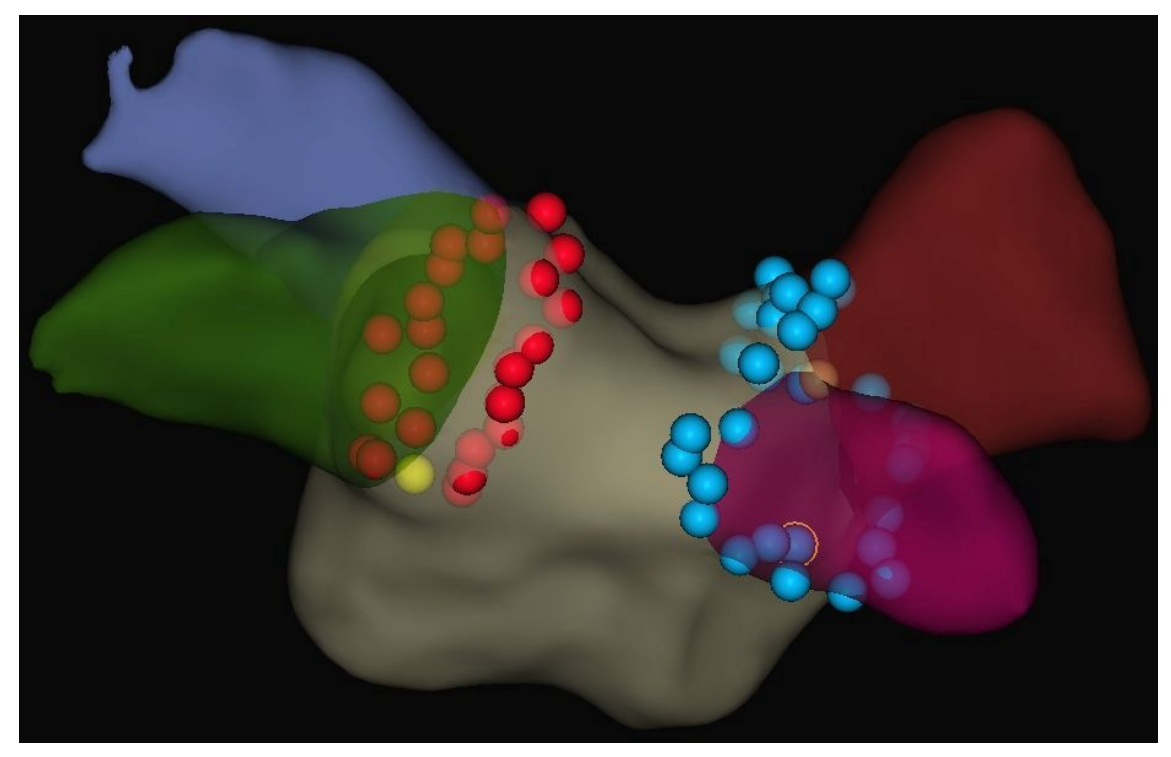

Comparison of RF Times Among Studies

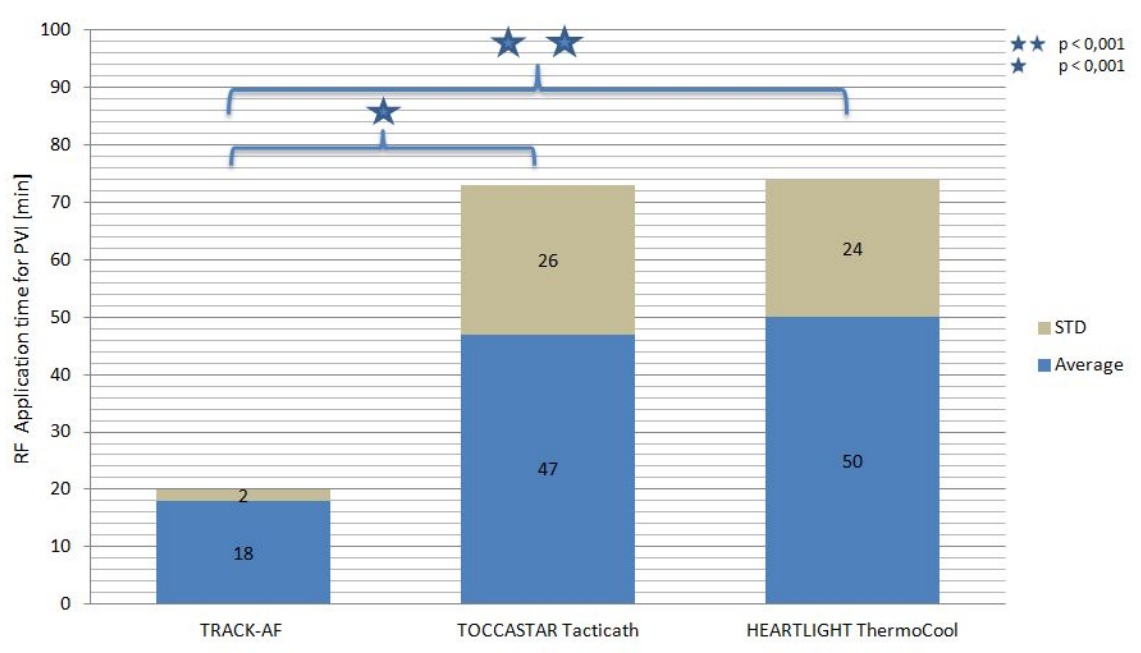

